\title{
On New Conservation Laws of Fin Equation
}

\author{
Gülden Gün Polat, ${ }^{1}$ Özlem Orhan, ${ }^{1}$ and Teoman Özer ${ }^{2}$ \\ ${ }^{1}$ Department of Mathematics, Faculty of Science and Letters, Istanbul Technical University, Maslak, 34469 Istanbul, Turkey \\ ${ }^{2}$ Division of Mechanics, Faculty of Civil Engineering, Istanbul Technical University, Maslak, 34469 Istanbul, Turkey \\ Correspondence should be addressed to Teoman Özer; tozer@itu.edu.tr
}

Received 12 April 2014; Accepted 17 June 2014; Published 14 August 2014

Academic Editor: Jian-Qiang Sun

Copyright (C) 2014 Gülden Gün Polat et al. This is an open access article distributed under the Creative Commons Attribution License, which permits unrestricted use, distribution, and reproduction in any medium, provided the original work is properly cited.

\begin{abstract}
We study the new conservation forms of the nonlinear fin equation in mathematical physics. In this study, first, Lie point symmetries of the fin equation are identified and classified. Then by using the relationship of Lie symmetry and $\lambda$-symmetry, new $\lambda$-functions are investigated. In addition, the Jacobi Last Multiplier method and the approach, which is based on the fact $\lambda$-functions are assumed to be of linear form, are considered as different procedures for lambda symmetry analysis. Finally, the corresponding new conservation laws and invariant solutions of the equation are presented.
\end{abstract}

\section{Introduction}

Fins are used in a large number of applications to increase the heat transfer from surfaces. Typically, the fin material has a high thermal conductivity. Due to having this property, it is very important in terms of technology. Generally, nonlinear heat conduction equation with fins is a mathematical model which can be represented by nonlinear differential equation. Pakdemirli and Sahin [1] obtained scaling, translational, and spiral group symmetries of the fin equation considered as a partial differential equation in which heat conduction coefficient is assumed to be function of temperature but the heat transfer coefficient is assumed to be only function of spatial variable. Bokhari et al. [2] investigated group theoretic analysis that provides different exact solutions or reduced equations specifically on traveling wave solutions and steady state type solutions. Vaneeva et al. [3] analyzed equivalence transformations and conditional equivalence groups and nonclassical symmetries of the fin equations are discussed in their study.

Lie point transformations are used for analyzing of differential equations mostly. These transformations leave the equation invariant which acts on the space of the dependent and independent variables. Revealing the symmetries of the equation by Lie group method perhaps enables us to obtain new solutions directly or from the known ones or via similarity reductions. In addition, the group classification of the differential equation based on the Lie point symmetries can be important in understanding the possible solutions of equations [4-12]. A part of this paper deals with Lie group analysis (symmetries, classification, and invariant solutions) of fin equation. Here, we analyze the special forms of thermal conductivity coeffcient $K(y)$ and the heat transfer coefficient $H(y)$.

For any second order ordinary differential equation by using different approaches $\lambda$-symmetries can be obtained directly. First studies based on this idea have been introduced by Muriel and Romero [13-16]. They have proved that under the invariance criteria obtained Lie symmetries enable deriving $\lambda$-symmetries in a direct way [13]. Moreover, they have demonstrated that integrating factors and first integrals can be determined algorithmically by making use of $\lambda$ symmetries. Another way obtaining $\lambda$-symmetry, for the sake of simplicity $\lambda$-function can be assumed in a linear form. As a result of this assumption the determining equation can be solved easily. In addition, in this study, we present the connection between Lie point symmetry and $\lambda$-symmetry to find nontrival $\lambda$-functions, corresponding integrating factors and first integrals.

The last approach to find $\lambda$-symmetry is based on the Jacobi last multiplier method which is presented by Nucci and Levi [17]. $\lambda$-symmetries and corresponding invariant 
solutions can be obtained by using the Jacobi last multiplier directly. This new method admits the new determining equation which includes $\lambda$-function that can be obtained from the divergence of the ordinary differential equation. $\lambda$ symmetries can be found from a new form of the prolongation formula which includes three unknown variables; therefore, the determining equations cannot be reduced to a simpler form. Despite this difficulty, we can reduce $\lambda$-function to two; the number of unknown functions by using the Jacobi last multiplier approach and the obtaining new functions called as infinitesimals functions can be evaluated simply. When all these reasons are taken into consideration, we examine $\lambda$-symmetries of the fin equation for different cases.

This study is organized as follows. In Section 2 we give some preliminaries on relationship for Lie symmetries and $\lambda$ symmetries. In Section 3 we introduce nonlinear fin equation and the corresponding determining equations. In Section 4, we present Lie symmetries of fin equation for different heat transfer coefficient and thermal conductivity. $\lambda$-symmetries, conservation laws and new reduced form of fin equation are obtained by using these Lie symmetries. In Section $5 \lambda$ symmetries in linear form are obtained and $\lambda$-symmetries based on Jacobi Last Multiplier method are considered as an alternative approach. Finally, In Section 6 we discuss some important results in the study.

\section{Relationship between Lie Point Symmetries and $\lambda$-Symmetries}

Let us consider the second order differential equation of the form

$$
\Delta\left(x, y^{(2)}\right)=0
$$

with $(x, y) \in M$, for some open subset $M \subset X \times Y \cong \mathbb{R}^{2}$. We denote by $M^{(k)}$ the corresponding $k$-jet space $M^{(k)} \subset X \times Y^{(k)}$, for $k \in \mathbb{N}$. Their elements are $\left(x, y^{(k)}\right)=\left(x, y, y_{1}, \ldots, y_{k}\right)$, where, for $1 \leq i \leq k, y_{i}$ denotes the derivative of order $i$ of $y$ with respect to $x$. We assume that the implicit function theorem can be applied to (1), and, as a consequence, that this equation can locally be written in the explicit form

$$
y^{\prime \prime}=\phi\left(x, y, y^{\prime}\right)
$$

and let vector field of (2) be in the form of

$$
A=\partial_{x}+y^{\prime} \partial_{y}+\phi\left(x, y, y^{\prime}\right) \partial_{y^{\prime}}
$$

Definition 1 (generalized prolongation formula). Let $X=$ $\xi(x, y) \partial_{x}+\eta(x, y) \partial_{y}$ be a vector field defined on $M$, and let $\lambda \in C^{\infty}\left(M^{(1)}\right)$ be an arbitrary function. The $\lambda$-prolongation of second order of $X$ denoted by $X^{[\lambda,(2)]}$ is the vector field defined on $M^{(2)}$ by

$$
X^{[\lambda,(2)]}=\xi(x, y) \frac{\partial}{\partial x}+\sum_{i=0}^{2} \eta^{[\lambda,(i)]}\left(x, y^{(i)}\right) \frac{\partial}{\partial y_{i}}
$$

where $\eta^{[\lambda,(0)]}=\eta(x, y)$ and

$$
\begin{aligned}
\eta^{[\lambda,(i)]}\left(x, y^{(i)}\right)= & D_{x}\left(\eta^{[\lambda,(i-1)]}\left(x, y^{(i-1)}\right)\right)-D_{x}(\xi(x, y)) y_{i} \\
& +\lambda\left(\eta^{[\lambda,(i-1)]}\left(x, y^{(i-1)}\right)-\xi(x, y) y_{i}\right),
\end{aligned}
$$

for $1 \leq i \leq 2$ where $D_{x}$ denotes the total derivative operator with respect to $x$.

The relationship between $\lambda$-symmetries, integrating factors and first integrals of second order differential equations is important from the mathematical point of view [13-16]. In terms of $A$ a first integral of (2) is any function in the form of $I\left(x, y, y^{\prime}\right)$ providing equality of $A(I)=0$. An integrating factor of (2) is any function satisfying the following equation:

$$
\mu\left[y^{\prime \prime}-\phi\left(x, y, y^{\prime}\right)\right]=D_{x} I
$$

Thus, $\lambda$-symmetries of second order differential equation (2) can be obtained directly by using Lie symmetries of this same equation. Secondly, let

$$
v=\xi(x, y) \frac{\partial}{\partial x}+\eta(x, y) \frac{\partial}{\partial y}
$$

be a Lie point symmetry of (2) and then the characteristic of $v$ is

$$
\mathrm{Q}=\eta-\xi y^{\prime}
$$

and thus the vector field $\partial_{y}$ is called $\lambda$-symmetry of (2) if the following equality:

$$
\lambda=\frac{A(\mathrm{Q})}{\mathrm{Q}}
$$

is satisfied.

If $\partial_{y}$ is assumed to be a $\lambda$-symmetry of (2) and $\omega\left(x, y, y^{\prime}\right)$ is a first order invariant of $X^{[\lambda,(1)]}$, namely, any particular solution of the equation

$$
\omega_{y}+\lambda\left(x, y, y^{\prime}\right) \omega_{y^{\prime}}=0
$$

then a first order invariant reduced equation of the form $\Delta_{R}\left(x, y, y^{\prime}\right)=0$ is obtained by using the reduction process associated to the $\lambda$-symmetry. Thus the general solution is found such as an equation of the implicit form:

$$
G(x, \omega)=C, \quad C \in \mathbb{R} .
$$

It is clear that $D_{x}\left(G\left(x, \omega\left(x, y, y^{\prime}\right)\right)\right)=0$ is an equivalent form of (2). Consequently,

$$
\mu\left(x, y, y^{\prime}\right)=G_{\omega}\left(x, y, \omega\left(x, y, y^{\prime}\right)\right) \cdot \omega_{x}\left(x, y, y^{\prime}\right)
$$

is an integrating factor of (2).

Theorem 2. Let $y^{(2)}=\phi\left(x, y^{(1)}\right)$ be a second order ordinary differential equation, where $\phi$ is an analytic function of its arguments. There exists a function $\lambda\left(x, y^{(k)}\right)$, for some $k<2$, such that the vector field $\partial_{y}$ is a $\lambda$-symmetry of the equation [15]. 


\section{Determining Equations for the Infinitesimal Symmetries}

The differential equation describing the nonlinear fin problem has been derived as follows:

$$
y^{\prime \prime}+\frac{K^{\prime}(y)}{K(y)}\left(y^{\prime}\right)^{2}-\frac{H(y)}{K(y)}=0,
$$

where $K(y)$ and $H(y)$ are thermal conductivity and heat transfer coefficient, respectively, which are considered as functions of temperature and $y=y(x)$ is the temperature function and $x$ is dimensional spatial variable [12].

If we consider an operator $X$ in the following form the nonlinear fin equation (13):

$$
X=\xi(x, y) \frac{\partial}{\partial x}+\eta(x, y) \frac{\partial}{\partial y}
$$

where $\xi(x, y)$ and $\eta(x, y)$ are infinitesimal functions. Here we consider second prolongation operator of (14) as in the following form:

$$
\begin{aligned}
X^{(2)}= & \xi(x, y) \frac{\partial}{\partial x}+\eta(x, y) \frac{\partial}{\partial y}+\eta_{1}\left(x, y, y_{1}\right) \frac{\partial}{\partial y_{1}} \\
& +\eta_{2}\left(x, y, y_{1}, y_{2}\right) \frac{\partial}{\partial y_{2}},
\end{aligned}
$$

since the highest derivative in (13) is second order in which $\eta_{1}$ and $\eta_{2}$ are defined:

$$
\begin{aligned}
\eta_{1}= & \eta_{x}+\left(\eta_{y}-\xi_{x}\right) y_{1}-\xi_{y} y_{1}^{2}, \\
\eta_{2}= & \eta_{x x}+\left(2 \eta_{x y}-\xi_{x x}\right) y_{1}+\left(\eta_{y y}-2 \xi_{x y}\right) y_{1}^{2} \\
& -\xi_{y y} y_{1}^{3}+\left(\eta_{y}-2 \xi_{x}\right) y_{2}-3 \xi_{y} y_{1} y_{2} .
\end{aligned}
$$

The application of (13) to (15) yields the invariance condition

$$
\begin{aligned}
X^{(2)}\left(y^{\prime \prime}\right. & +\frac{K^{\prime}(y)}{K(y)}\left(y^{\prime}\right)^{2} \\
& \left.-\frac{H(y)}{K(y)}\right)\left.\right|_{y^{\prime \prime}=-\left(K^{\prime}(y) / K(y)\right)\left(y^{\prime}\right)^{2}+H(y) / K(y)},
\end{aligned}
$$

or equivalently

$$
\begin{aligned}
\eta_{x x} & +y_{1}\left(-\xi_{x x}+2 \eta_{x y}\right)+y_{1}^{2}\left(-2 \xi_{x y}+\eta_{y y}\right)-y_{1}^{3} \xi_{y y} \\
& +\left(-\frac{K^{\prime}(y)}{K(y)} y_{1}^{2}+\frac{H(y)}{K(y)}\right)\left(-2 \xi_{x}+\eta_{y}\right) \\
& +y_{1}\left(-\frac{K^{\prime}(y)}{K(y)} y_{1}^{2}+\frac{H(y)}{K(y)}\right)\left(-3 \xi_{y}\right) \\
+ & 2\left(\eta_{x}+y_{1}\left(\eta_{y}-\xi_{x}\right)-y_{1}^{2} \xi_{y}\right) \frac{K^{\prime}(y)}{K(y)} y_{1} \\
+ & \eta \frac{K^{\prime \prime}(y) K(y)-K^{\prime}(y)^{2}}{K^{2}(y)} y_{1}^{2} \\
+ & \eta \frac{H(y) K^{\prime}(y)-H^{\prime}(y) K(y)}{K^{2}(y)}=0 .
\end{aligned}
$$

In order to obtain the determining equations, the equation (19) can be separated with respect to $y_{1}$ and its powers:

$$
\begin{gathered}
-\xi_{y y}+\frac{K^{\prime}(y)}{K(y)} \xi_{y}=0 \\
-2 \xi_{x y}+\eta_{y y}+\frac{K^{\prime}(y)}{K(y)} \eta_{y}+\frac{K^{\prime \prime}(y) K(y)-K^{\prime}(y)^{2}}{K^{2}(y)} \eta=0 \\
-\xi_{x x}+2 \eta_{x y}-3 \frac{H(y)}{K(y)} \xi_{y}+2 \frac{K^{\prime}(y)}{K(y)} \eta_{x}=0 \\
\eta_{x x}-2 \frac{H(y)}{K(y)} \xi_{x}+\frac{H(y)}{K(y)} \eta_{y} \\
+\frac{H(y) K^{\prime}(y)-H^{\prime}(y) K(y)}{K^{2}(y)} \eta=0
\end{gathered}
$$

\section{Lie Symmetries and Corresponding $\lambda$-Symmetries}

In this section, the aim of our study is to get Lie point symmetries, corresponding lambda symmetries, conserved forms and invariant solutions by using different $K(y)$ and $H(y)$ functions. To find the infinitesimals, (20)-(23) should be solved together. First the equation (20) is integrated:

$$
\xi(x, y)=a(x) \int K(y) d y+b(x),
$$

and integrating (21) with respect to $y$ and solving for $\eta$ we have

$$
\begin{aligned}
\eta(x, y)=( & \left(2 b^{\prime}(x)+c^{\prime}(x)\right) \int K(y) d y \\
& \left.+a^{\prime}(x) \int K(y)\left(\int K(y) d y\right) d y\right) \\
& \times(K(y))^{-1}+\frac{d(x)}{K(y)} .
\end{aligned}
$$


The infinitesimals $\xi$ and $\eta$ are inserted into (22), and the heat transfer coefficient is calculated in the following form:

$$
h(y)=\frac{a^{\prime \prime}(x) \int K(y) d y+3 b^{\prime \prime}(x)+2 c^{\prime \prime}(x)}{3 a(x)},
$$

in which the equation (26) leads to that heat transfer coefficient $H(y)$ can be obtained by examining different cases of thermal conductivity $K(y)$.

4.1. Constant Thermal Conductivity: $K(y)=k_{0}$. Firstly we consider a constant thermal conductivity. Substituting $K(y)=k_{0}$ in (26) we can write

$$
h(y)=\frac{k_{0} a^{\prime \prime}(x)}{3 a(x)} y+\frac{3 b^{\prime \prime}(x)+2 c^{\prime \prime}(x)}{3 a(x)},
$$

and in (16) we see that the heat transfer coefficient $H(y)$ is in the linear form. By defining $\tau_{1}$ and $\tau_{2}$ such that

$$
\tau_{1}=\frac{k_{0} a^{\prime \prime}(x)}{3 a(x)}, \quad \tau_{2}=\frac{3 b^{\prime \prime}(x)+2 c^{\prime \prime}(x)}{3 a(x)},
$$

then, rewriting (16) with (17) the relation

$$
H(y)=\tau_{1} y+\tau_{2},
$$

is obtained in which $\tau_{1}$ and $\tau_{2}$ are constants. To classify the results systematically, we consider the following subcases: (i) $\tau_{1}=\tau_{2}=0$, (ii) $\tau_{1}=0, \tau_{2} \neq 0$, (iii) $\tau_{1} \neq 0, \tau_{2}=0$, and (iv) $\tau_{1} \neq 0, \tau_{2} \neq 0$.

Case 1 (if $\tau_{1}=0, \tau_{2}=0$ ). The fin equation (13) is

$$
y^{\prime \prime}=0 \text {. }
$$

For this case (20)-(23) finally yield

$$
\begin{aligned}
& \xi(x, y)=c_{6}+x\left(c_{7}+c_{8} x\right)+\left(c_{4}+c_{5} x\right) y, \\
& \eta(x, y)=c_{1}+y\left(c_{3}+c_{5} y\right)+x\left(c_{2}+c_{8} y\right),
\end{aligned}
$$

where $c_{i}, i=1, \ldots, 8$ are constants. The algebra consists of an eight-parameter finite Lie group of transformations [4].

If parameter $c_{5}$ is selected and the remaining ones are set to be zero, the infinitesimals $\xi$ and $\eta$ are

$$
\xi=x y, \quad \eta=y^{2} .
$$

Therefore, the characteristic is written:

$$
\mathrm{Q}=y^{2}-x y y^{\prime}
$$

By using (9), we obtain the $\lambda$-symmetry

$$
\lambda=\frac{y^{\prime}}{y} .
$$

A solution of (10) for this case is

$$
w\left(x, y, y^{\prime}\right)=\frac{y^{\prime}}{y}
$$

and we can write $w=y^{\prime} / y$; then to obtain fin equation in terms of $\left\{x, w, w^{\prime}\right\}$ one can write

$$
y^{\prime}=w y, \quad y^{\prime \prime}=w^{2} y+y w^{\prime}
$$

By using these equalities (36) we find the following equation:

$$
w^{\prime}(x)+w^{2}(x)=0
$$

in which the general solution is

$$
w(x)=\frac{1}{x-G}, \quad G \in \mathbb{R}
$$

and then the integrating factor becomes

$$
\mu=\frac{y}{y^{\prime 2}} .
$$

Conservation law is

$$
D_{x}\left(x-\frac{y(x)}{y^{\prime}(x)}\right)=0,
$$

and the invariant solution is

$$
y(x)=\alpha x,
$$

where $\alpha$ is a constant.

Case $2\left(\tau_{1}=0, \tau_{2} \neq 0\right)$. The fin equation becomes

$$
y^{\prime \prime} k_{0}-\tau_{2}=0,
$$

and the solutions of (20)-(23) yield an eight-parameter Lie group of transformations:

$$
\begin{aligned}
\xi(x, y)= & \left(k _ { 0 } \left(2\left(c_{1}-c_{7}-4 c_{8} x\right) y k_{0}\right.\right. \\
& +\left(c_{3}+c_{5}+\left(c_{2}+2 c_{6}\right) x\right. \\
& \left.\left.\left.+\left(c_{1}+3 c_{7}\right) x^{2}+4 c_{8} x^{3}\right) \tau_{2}\right)\right) \times\left(2 \tau_{2}^{2}\right)^{-1}, \\
\eta(x, y)= & \left(-4 c_{8} y^{2} k_{0}^{2}+\left(c_{2}+2 c_{1} x\right) y k_{0} \tau_{2}\right. \\
& \left.+\left(c_{4}+x\left(c_{5}+x\left(c_{6}+x\left(c_{7}+c_{8} x\right)\right)\right)\right) \tau_{2}^{2}\right) \\
& \times\left(\tau_{2}^{2}\right)^{-1},
\end{aligned}
$$

where $c_{i}, i=1, \ldots, 8$ are constants.

For the selection of parameter $c_{5}$, the infinitesimals are

$$
\xi=\frac{k_{0}}{2 \tau_{2}}, \quad \eta=x .
$$

By using (9), the $\lambda$-symmetry yields

$$
\lambda=\frac{\tau_{2}}{2 x \tau_{2}-y^{\prime} k_{0}} .
$$


A solution of (10) is equal to

$$
w\left(x, y, y^{\prime}\right)=\frac{\tau_{2}}{2 x \tau_{2}-y^{\prime} k_{0}},
$$

and reduced form becomes

$$
w^{\prime}(x)+w^{2}(x)=0
$$

in which the general solution is

$$
w(x)=\frac{1}{x-G}, \quad G \in \mathbb{R},
$$

and the integrating factor is

$$
\mu=\frac{k_{0}}{\tau_{2}} .
$$

Conservation law is found as

$$
D_{x}\left(\frac{y^{\prime}(x) k_{0}}{\tau_{2}}-x\right)=0,
$$

and the invariant solution is

$$
y(x)=\alpha+\frac{\beta x \tau_{2}}{k_{0}}+\frac{x^{2} \tau_{2}}{2 k_{0}},
$$

where $\alpha$ and $\beta$ are constants.

Case 3 (if $\tau_{1} \neq 0, \tau_{2}=0$ ). Equation (13) is equal to

$$
y^{\prime \prime} k_{0}-y \tau_{1}=0
$$

and the infinitesimals for this equation are

$$
\begin{aligned}
& \xi(x, y)=e^{-2 x \sqrt{\tau_{1}} / \sqrt{k_{0}}}\left(c_{7}+e^{4 x \sqrt{\tau_{1}} / \sqrt{k_{0}}} c_{8}+e^{x \sqrt{\tau_{1}} / \sqrt{k_{0}}} c_{1} y\right. \\
& \left.+e^{3 x \sqrt{\tau_{1}} / \sqrt{k_{0}}} c_{2} y\right)+\frac{c_{6} k_{0}}{2 \tau_{1}}, \\
& \eta(x, y)=\frac{1}{2 \sqrt{k_{0}}} e^{-2 x \sqrt{\tau_{1}} / \sqrt{k_{0}}} \\
& \times\left(e ^ { x \sqrt { \tau _ { 1 } } / \sqrt { k _ { 0 } } } \left(2 c_{3}+2 e^{2 x \sqrt{\tau_{1}} / \sqrt{k_{0}}} c_{4}\right.\right. \\
& \left.+e^{x \sqrt{\tau_{1}} / \sqrt{k_{0}}} c_{5} y\right) \sqrt{k_{0}} \\
& +2 y\left(-c_{7}+e^{4 x \sqrt{\tau_{1}} / \sqrt{k_{0}}} c_{8}-e^{x \sqrt{\tau_{1}} / \sqrt{k_{0}}} c_{1} y\right. \\
& \left.\left.+e^{3 x \sqrt{\tau_{1}} / \sqrt{k_{0}}} c_{2} y\right) \sqrt{\tau_{1}}\right)
\end{aligned}
$$

where $c_{i}, i=1, \ldots, 8$ are constants.

For selection of parameter $c_{1}$ infinitesimals are found:

$$
\xi=e^{x \sqrt{\tau_{1}} / \sqrt{k_{0}}} y, \quad \eta=-\frac{e^{x \sqrt{\tau_{1}} / \sqrt{k_{0}}} y^{2} \tau_{1}}{\sqrt{k_{0}}} .
$$

By using (9) we obtain the $\lambda$-symmetry

$$
\lambda=\frac{y^{\prime}}{y} .
$$

A solution of (10) for this case is

$$
w\left(x, y, y^{\prime}\right)=\frac{y^{\prime}}{y},
$$

and reduced form is

$$
w^{\prime}(x)+w^{2}(x)-\frac{\tau_{1}}{k_{0}}=0,
$$

in which the general solution is

$$
w(x)=\sqrt{\frac{\tau_{1}}{k_{0}}} \tanh \left(\sqrt{\frac{\tau_{1}}{k_{0}}}\left(x-G k_{0}\right)\right), \quad G \in \mathbb{R},
$$

and then the integrating factor becomes

$$
\mu=\frac{y}{y^{\prime 2} k_{0}-y^{2} \tau_{1}} .
$$

Conservation law is

$$
D_{x}\left(\frac{1}{k_{0} \sqrt{\tau_{1}}}\left(x \sqrt{\tau_{1}}-\operatorname{arctanh}\left(\frac{k_{0}}{\sqrt{\tau_{1}}} \frac{y^{\prime}(x)}{y(x)}\right)\right)\right)=0,
$$

and the invariant solution is

$$
y(x)=\alpha \cosh \left(\sqrt{\frac{\tau_{1}}{k_{0}}}\left(x-\beta k_{0}\right)\right),
$$

where $\alpha$ and $\beta$ are constants.

Case $4\left(\tau_{1} \neq 0, \tau_{2} \neq 0\right)$. In this case we obtain (13)

$$
k_{0} y^{\prime \prime}-\tau_{1} y-\tau_{2}=0 .
$$

Infinitesimals are found as follows:

$$
\begin{aligned}
& \xi(x, y)=\frac{1}{3 \sqrt{\tau_{1}}}\left(e^{-2 x \sqrt{\tau_{1}} / \sqrt{k_{0}}}\left(c_{3}-c_{4} e^{4 x \sqrt{\tau_{1}} / \sqrt{k_{0}}}\right)\right. \\
&\left.\times \sqrt{k_{0}}+c_{1} \sqrt{\tau_{1}}\right), \\
& \eta(x, y)=\frac{1}{3 k_{0} \tau_{1}} e^{-2 x \sqrt{\tau_{1}} / \sqrt{k_{0}}} \\
& \times\left(6 \tau_{1} e^{x \sqrt{\tau_{1}} / \sqrt{k_{0}}}\right. \\
& \quad \times\left(c_{5}+c_{6} e^{2 x \sqrt{\tau_{1}} / \sqrt{k_{0}}}\right)-k_{0}\left(\tau_{1} y+\tau_{2}\right) \\
&\left.\quad \times\left(2 c_{3}-9 c_{2} e^{-2 x \sqrt{\tau_{1}} / \sqrt{k_{0}}}+2 c_{4} e^{-2 x \sqrt{\tau_{1}} / \sqrt{k_{0}}}\right)\right),
\end{aligned}
$$

where $c_{i}, i=1, \ldots, 6$ are constants. 
For selection of parameter $c_{1}$ infinitesimals are found:

$$
\xi=\frac{e^{-2 x \sqrt{\tau_{1}} / \sqrt{k_{0}}} \sqrt{k_{0}}}{3 \sqrt{\tau_{1}}}, \quad \eta=e^{-2 x \sqrt{\tau_{1}} / \sqrt{k_{0}}} \frac{\left(\tau_{1} y+\tau_{2}\right)}{3 \tau_{1}} .
$$

By using (9) we obtain the $\lambda$-symmetry

$$
\lambda=-\sqrt{\frac{\tau_{1}}{k_{0}}} .
$$

A solution of (10) for this case is

$$
w\left(x, y, y^{\prime}\right)=y^{\prime}+y \frac{\tau_{1}}{k_{0}},
$$

and reduced form is equal to

$$
w^{\prime}(x)+w(x) \sqrt{\frac{\tau_{1}}{k_{0}}}-\frac{\tau_{2}}{k_{0}}=0,
$$

in which the general solution is

$$
w(x)=e^{x \sqrt{\tau_{1}} / \sqrt{k_{0}}} G-\frac{\tau_{2}}{\sqrt{k_{0} \tau_{1}}}, \quad G \in \mathbb{R},
$$

and the integrating factor yields

$$
\mu=e^{-x \sqrt{\tau_{1}} / \sqrt{k_{0}}} .
$$

Conservation law is

$$
D_{x}\left(\frac{e^{-x \sqrt{\tau_{1}} / \sqrt{k_{0}}}}{\sqrt{k_{0} \tau_{1}}}\left(y^{\prime}(x) \sqrt{k_{0} \tau_{1}}+y(x) \tau_{1}+\tau_{2}\right)\right)=0,
$$

and the invariant solution is

$$
y(x)=\alpha e^{-x \sqrt{\tau_{1}} / \sqrt{k_{0}}}+\frac{e^{x \sqrt{\tau_{1}} / \sqrt{k_{0}}} \beta \sqrt{k_{0}}}{\sqrt{\tau_{1}}}-\frac{\tau_{2}}{\tau_{1}},
$$

where $\alpha$ and $\beta$ are constants.

4.2. Linear Thermal Conductivity $K(y)=k_{0} y$. If $K(y)=$ $k_{0} y$ inserted into (26), and then the heat transfer coefficient is obtained in the following form:

$$
H(y)=\frac{k_{0} a^{\prime \prime}(x)}{6 a(x)} y^{2}+\frac{3 b^{\prime \prime}(x)+2 c^{\prime \prime}(x)}{3 a(x)} .
$$

Let us consider the form of $\tau_{1}$ and $\tau_{2}$ such as is

$$
\tau_{1}=\frac{k_{0} a^{\prime \prime}(x)}{6 a(x)}, \quad \tau_{2}=\frac{3 b^{\prime \prime}(x)+2 c^{\prime \prime}(x)}{3 a(x)} .
$$

Then,

$$
H(y)=\tau_{1} y^{2}+\tau_{2}
$$

To classify the results systematically, we consider the following subcases: (i) $\tau_{1}=\tau_{2}=0$, (ii) $\tau_{1}=0, \tau_{2} \neq 0$, (iii) $\tau_{1} \neq$ $0, \tau_{2}=0$, and (iv) $\tau_{1} \neq 0, \tau_{2} \neq 0$.
Case 1 (if $\tau_{1}=0, \tau_{2}=0$ ). Then the equation is

$$
y^{\prime \prime} y+y^{\prime 2}=0,
$$

and the solution of (20)-(23) gives to the infinitesimals

$$
\begin{gathered}
\xi(x, y)=\frac{1}{2} y^{2} c_{1}+c_{2}+c_{3} x-\frac{2}{3}\left(c_{4} x+c_{5} x^{2}\right), \\
\eta(x, y)=\frac{1}{6}\left(6 c_{3}-c_{4}-2 c_{5} x\right) y
\end{gathered}
$$

where $c_{i}, i=1, \ldots, 8$ are constants.

If parameter $c_{5}$ is selected and the remaining ones are set to be zero, the infinitesimals $\xi$ and $\eta$ are

$$
\xi=-\frac{2}{3} x^{2}, \quad \eta=-\frac{x y}{3} .
$$

By using (9) we obtain the $\lambda$-symmetry

$$
\lambda=\frac{y-y^{\prime}}{x y} .
$$

A solution of (10) for this case is

$$
w\left(x, y, y^{\prime}\right)=\frac{y\left(2 x y y^{\prime}-y\right)}{2 x},
$$

and reduced form is found as below:

$$
w^{\prime}(x) x+w(x)=0,
$$

in which the general solution is

$$
w(x)=\frac{G}{x}, \quad G \in \mathbb{R},
$$

and the integrating factor becomes as the form

$$
\mu=x y .
$$

Conservation law is

$$
D_{x}\left(-\frac{1}{2} y(x)\left(y(x)-2 x y^{\prime}(x)\right)\right)=0,
$$

and the invariant solution is

$$
y(x)=-\sqrt{e^{2 \alpha} x-2 \beta},
$$

where $\alpha$ and $\beta$ are constants.

Case $2\left(\tau_{1}=0, \tau_{2} \neq 0\right)$. For this case we obtain

$$
y^{\prime \prime} y k_{0}+y^{\prime 2} k_{0}-\tau_{2}=0,
$$


then the infinitesimals (24) and (25) are

$$
\begin{gathered}
\xi(x, y)=\left(k _ { 0 } \left(\left(c_{1}-c_{7}-4 c_{8} x\right) y^{2} k_{0}\right.\right. \\
+\left(c_{3}+c_{5}+\left(c_{2}+2 c_{6}\right) x\right. \\
\left.\left.\left.+\left(c_{1}+3 c_{7}\right) x^{2}+4 c_{8} x^{3}\right) \tau_{2}\right)\right) \\
\times\left(2 \tau_{2}^{2}\right)^{-1}, \\
\eta(x, y)=\left(-2 c_{8} y^{4} k_{0}^{2}+\left(c_{2}+2 c_{1} x\right) y^{2} k_{0} \tau_{2}\right. \\
\left.+2\left(c_{4}+x\left(c_{5}+x\left(c_{6}+x\left(c_{7}+c_{8} x\right)\right)\right)\right) \tau_{2}^{2}\right) \\
\times\left(2 y \tau_{2}^{2}\right)^{-1},
\end{gathered}
$$

where $c_{i}, i=1, \ldots, 8$ are constants.

If parameter $c_{3}$ is selected and the remaining ones are set to be zero, the infinitesimals $\xi$ and $\eta$ are

$$
\xi=-\frac{k_{0}}{2 \tau_{2}}, \quad \eta=0
$$

By using (9) we obtain the $\lambda$-symmetry

$$
\lambda=\frac{\tau_{2}-y^{\prime 2} k_{0}}{y y^{\prime} k_{0}} .
$$

A solution of (10) for this case is

$$
w\left(x, y, y^{\prime}\right)=\frac{1}{2 k_{0}} \ln \left(y^{2}\left(y^{\prime 2} k_{0}-\tau_{2}\right)\right)
$$

and reduced form is

$$
w^{\prime}(x)=0,
$$

in which the general solution is

$$
w(x)=G, \quad G \in \mathbb{R},
$$

and then the integrating factor becomes

$$
\mu=\frac{y^{\prime}}{\tau_{2}-y^{\prime 2} k_{0}} .
$$

Conservation law is

$$
D_{x}\left(\frac{1}{2 k_{0}} \ln \left(y(x)^{2}\left(y^{\prime}(x)^{2} k_{0}-\tau_{2}\right)\right)\right)=0,
$$

and the invariant solution is

$$
y(x)=\mp \frac{1}{\tau_{2}}-\sqrt{e^{2 \alpha k_{0}}+\beta^{2} \tau_{2}^{2}+\frac{x^{2} \tau_{2}}{k_{0}}-\frac{2 x \beta \tau_{2}^{2}}{\sqrt{k_{0}}}},
$$

where $\alpha$ and $\beta$ are constants.
Case 3. For the choice of $\tau_{1} \neq 0, \tau_{2}=0$ the fin equation (13) becomes

$$
y^{\prime \prime} y k_{0}+y^{\prime 2} k_{0}-y^{2} \tau_{1}=0 .
$$

After some manipulations equations (20)-(23) yield

$$
\begin{aligned}
& \xi(x, y)= e^{-2 x \sqrt{2 \tau_{1}} / \sqrt{k_{0}}} c_{7}+e^{2 x \sqrt{2 \tau_{1}} / \sqrt{k_{0}}} c_{8} \\
&+\frac{1}{2} e^{-x \sqrt{2 \tau_{1}} / \sqrt{k_{0}}}\left(c_{1}+e^{2 x \sqrt{2 \tau_{1}} / \sqrt{k_{0}}} c_{2}\right) y^{2} \\
&+\frac{c_{6} k_{0}}{2 \tau_{1}}, \\
& \eta(x, y)= \frac{1}{4 y \sqrt{k_{0}}} e^{-2 x \sqrt{2 \tau_{1}} / \sqrt{k_{0}}} \\
& \times\left(e ^ { x \sqrt { 2 \tau _ { 1 } } / \sqrt { k _ { 0 } } } \left(4 c_{3}+4 e^{2 x \sqrt{2 \tau_{1}} / \sqrt{k_{0}}} c_{4}\right.\right. \\
&+\sqrt{2} y^{2}\left(-2 c_{7}+2 e^{4 x \sqrt{2 \tau_{1}} / \sqrt{k_{0}}} c_{5} y^{2}\right) \sqrt{k_{0}} \\
& \quad-e^{x \sqrt{2 \tau_{1}} / \sqrt{k_{0}}} c_{1} y^{2} \\
&\left.\left.+e^{3 x \sqrt{2 \tau_{1}} / \sqrt{k_{0}}} c_{2} y^{2}\right) \sqrt{\tau_{1}}\right),
\end{aligned}
$$

where $c_{i}, i=1, \ldots, 8$ are constants.

For selection of parameter $c_{3}$ infinitesimals are found:

$$
\xi=0, \quad \eta=\frac{1}{y} e^{-x \sqrt{2 \tau_{1}} / \sqrt{k_{0}}} .
$$

By using (9) we obtain the $\lambda$-symmetry

$$
\lambda=-\frac{y^{\prime} \sqrt{k_{0}}+y \sqrt{2 \tau_{1}}}{y \sqrt{k_{0}}} .
$$

A solution of (10) for this case is

$$
w\left(x, y, y^{\prime}\right)=\frac{y}{2 \sqrt{k_{0}}}\left(2 y^{\prime} \sqrt{k_{0}}+y \sqrt{2 \tau_{1}}\right),
$$

and reduced form is

$$
w^{\prime}(x)-\sqrt{\frac{2 \tau_{1}}{k_{0}}} w(x)=0,
$$

in which the general solution is

$$
w(x)=e^{x \sqrt{2 \tau_{1}} / \sqrt{k_{0}}} G, \quad G \in \mathbb{R},
$$

and the integrating factor becomes

$$
\mu=e^{-x \sqrt{2 \tau_{1}} / \sqrt{k_{0}}} y .
$$

Conservation law is

$$
D_{x}\left(\frac{e^{-x \sqrt{2 \tau_{1}} / \sqrt{k_{0}}} y(x)}{2 \sqrt{k_{0}}}\left(2 y^{\prime}(x) \sqrt{k_{0}}+\sqrt{2 \tau_{1}} y(x)\right)\right)=0,
$$


and the invariant solution is

$$
y(x)=\mp \sqrt{e^{-x \sqrt{2 \tau_{1}} / \sqrt{k_{0}}} \alpha+\frac{\beta \sqrt{k_{0}}}{\sqrt{2 \tau_{1}}} e^{x \sqrt{2 \tau_{1}} / \sqrt{k_{0}}}},
$$

where $\alpha$ and $\beta$ are constants.

Case $4\left(\tau_{1} \neq 0, \tau_{2} \neq 0\right)$. For this case, $(13)$ is

$$
y^{\prime \prime} y k_{0}+y^{\prime 2} k_{0}-y^{2} \tau_{1}-\tau_{2}=0,
$$

and infinitesimals are

$$
\xi(x, y)=c_{1}, \quad \eta(x, y)=0,
$$

where $c_{1}$ is constant. This result corresponds to principal Lie algebra which is a one-parameter finite Lie group of transformations.

By applying (9) we derive the $\lambda$-symmetry

$$
\lambda=\frac{y^{2} \tau_{1}-y^{\prime 2} k_{0}+\tau_{2}}{y y^{\prime} k_{0}} .
$$

A solution of (10) can be obtained:

$$
w\left(x, y, y^{\prime}\right)=\frac{2 y^{2} y^{\prime 2} k_{0}-y^{4} \tau_{1}-2 y^{2} \tau_{2}}{2 k_{0}},
$$

and the reduced form is

$$
w^{\prime}(x)=0,
$$

in which the general solution is found

$$
w(x)=G, \quad G \in \mathbb{R},
$$

and the integrating factor can be derived as below:

$$
\mu=2 y^{2} y^{\prime} \text {. }
$$

Conservation law is

$$
D_{x}\left(\frac{1}{2 k_{0}}\left(2 y(x)^{2} y^{\prime}(x)^{2} k_{0}-y(x)^{4} \tau_{1}-2 y(x)^{2} \tau_{2}\right)\right)=0 .
$$

4.3. $K(y)=k_{0} y^{n}, n \neq-1,0$. If $K(y)=k_{0} y^{n}$ is inserted into (26), then we have

$$
H(y)=\frac{k_{0} a^{\prime \prime}(x)}{3(n+1) a(x)} y^{n+1}+\frac{3 b^{\prime \prime}(x)+2 c^{\prime \prime}(x)}{3 a(x)} .
$$

By defining

$$
\tau_{1}=\frac{k_{0} a^{\prime \prime}(x)}{3(n+1) a(x)}, \quad \tau_{2}=\frac{3 b^{\prime \prime}(x)+2 c^{\prime \prime}(x)}{3 a(x)},
$$

then, $H(y)$ is rewritten in the form

$$
H(y)=\tau_{1} y^{n+1} \tau_{2} .
$$

To classify the results systematically, we consider the following subcases: (i) $\tau_{1}=\tau_{2}=0$, (ii) $\tau_{1}=0, \tau_{2} \neq 0$, (iii) $\tau_{1} \neq$ $0, \tau_{2}=0$, and (iv) $\tau_{1} \neq 0, \tau_{2} \neq 0$.
Case $1\left(\tau_{1}=0, \tau_{2}=0\right)$. The fin equation is

$$
y^{\prime \prime} y+n y^{\prime 2}=0
$$

The solution of (20)-(23) gives to an eight-parameter Lie group of transformations:

$$
\begin{aligned}
\xi(x, y)= & \left(c_{4}(n+1)\right. \\
& +c_{1}(n+1)^{2} x^{2}+c_{3} y^{(n+1)} \\
& \left.+(n+1) x\left(c_{5}+c_{8}(n+1) y^{n+1}\right)\right) \\
& \times(n+1)^{-1}, \\
\eta(x, y)=c_{7} y & +c_{1} x y+c_{6} y^{-n}+c_{2} x y^{-n}+c_{8} y^{n+2},
\end{aligned}
$$

where $c_{i}, i=1, \ldots, 8$ are constants

For selection of parameter $c_{1}$ infinitesimals are found:

$$
\xi=(n+1) x^{2}, \quad \eta=x y .
$$

By using (9) we obtain the $\lambda$-symmetry

$$
\lambda=\frac{y-n y^{\prime}}{x y} .
$$

A solution of (10) for this case is

$$
w\left(x, y, y^{\prime}\right)=\frac{y^{n}}{(n+1) x}\left(x y^{\prime}-y+n x y^{\prime}\right),
$$

and reduced form is equal to

$$
x w^{\prime}(x)+w(x)=0,
$$

in which the general solution yields

$$
w(x)=\frac{G}{x}, \quad G \in \mathbb{R},
$$

and then the integrating factor becomes

$$
\mu=y^{n} x
$$

Conservation law is

$$
D_{x}\left(\frac{y(x)^{n}}{n+1}\left(n x y^{\prime}(x)+x y^{\prime}(x)-y(x)\right)\right)=0,
$$

and the invariant solution is

$$
y(x)=\left(-\alpha-\alpha n+e^{\beta(n+1)} x+e^{\beta(n+1)} n x\right)^{1 /(n+1)},
$$

where $\alpha$ and $\beta$ are constants.

Case $2\left(n \neq-1 \lambda_{1}=0, \lambda_{2} \neq 0\right)$. For this case (13) equals

$$
\begin{gathered}
y^{\prime \prime} y^{n+1} k_{0}+n y^{n} y^{\prime 2} k_{0}-y \tau_{2}=0, \\
\xi(x, y)=\frac{1}{6(n+1)}\left(6 c_{1} y^{n+1} k_{0}+(n+1)\right. \\
\left.\times\left(6 c_{3}+6 c_{2} x+3 c_{1} x^{2} \tau_{2}\right)\right), \\
\eta(x, y)=\frac{2 y}{n+1}\left(c_{2}+c_{1} x \tau_{2}\right),
\end{gathered}
$$

where $c_{i}, i=1, \ldots, 4$ are constants. 
For selection of parameter $c_{3}$ infinitesimals are found:

$$
\xi=1, \quad \eta=0 .
$$

By using (9) we obtain the $\lambda$-symmetry

$$
\lambda=\frac{y^{(1-n)} \tau_{2}-n y^{\prime 2} k_{0}}{y y^{\prime} k_{0}} .
$$

A solution of (10) for this case is

$$
w\left(x, y, y^{\prime}\right)=\frac{y^{n}\left(y^{n} y^{\prime 2} k_{0}(n+1)-2 y \tau_{2}\right)}{(n+1) k_{0}},
$$

and reduced form is

$$
w^{\prime}(x)=0,
$$

in which the general solution is

$$
w(x)=G, \quad G \in \mathbb{R},
$$

and then the integrating factor becomes

$$
\mu=2 y^{2 n} y^{\prime}
$$

Conservation law is

$$
D_{x}\left(\frac{y(x)^{n}\left(y(x)^{n} y^{\prime}(x)^{2} k_{0}(n+1)-2 y(x) \tau_{2}\right)}{(n+1) k_{0}}\right)=0 .
$$

Case $3\left(\tau_{1} \neq 0, \tau_{2}=0\right)$. In this case fin equation becomes

$$
y^{\prime \prime} y^{n+1} k_{0}+n y^{n} y^{\prime 2} k_{0}-\tau_{1}=0,
$$

and infinitesimals functions are found as below

$$
\begin{gathered}
\xi(x, y)=c_{1}+\frac{e^{-2 x \sqrt{(n+1) \tau_{1}} / \sqrt{k_{0}}} \sqrt{k_{0}}}{3 \sqrt{(n+1) \tau_{1}}} \\
\times\left(c_{3}-c_{4} e^{4 x \sqrt{(n+1) \tau_{1}} / \sqrt{k_{0}}}\right), \\
\eta(x, y)=\frac{y}{6(n+1)}\left(9 c_{2}-2 e^{-2 x \sqrt{(n+1) \tau_{1}} / \sqrt{k_{0}}}\right. \\
\left.\times\left(c_{3}+c_{4} e^{4 x \sqrt{(n+1) \tau_{1}} / \sqrt{k_{0}}}\right)\right),
\end{gathered}
$$

where $c_{i}, i=1, \ldots, 2$ are constants.

For selection of parameter $c_{2}$ infinitesimals are found

$$
\xi=0, \quad \eta=\frac{3 y}{2(n+1)},
$$

equation (9) gives the $\lambda$-symmetry

$$
\lambda=\frac{y^{\prime}}{y} \text {. }
$$

For this case the solution of (10) is

$$
w\left(x, y, y^{\prime}\right)=\frac{y^{\prime}}{y}
$$

and reduced form can be written as

$$
\left(w^{\prime}(x)+(n+1) w(x)^{2}\right) k_{0}-\tau_{1}=0,
$$

in which the general solution is

$$
\begin{array}{r}
w(x)=\frac{\tau_{1} \tanh }{\sqrt{(n+1) k_{0}}}\left(x \sqrt{\frac{\tau_{1}(n+1)}{k_{0}}}-G \sqrt{\tau_{1}(n+1) k_{0}}\right) \\
G \in \mathbb{R},
\end{array}
$$

and the integrating factor is found as below:

$$
\mu=\frac{y}{(n+1) y^{\prime 2} k_{0}-y^{2} \tau_{1}} .
$$

Conservation law is

$$
D_{x}\left(\frac{x}{k_{0}}-\frac{1}{\sqrt{(n+1) k_{0} \tau_{1}}} \operatorname{arctanh}\left(\frac{y^{\prime}}{y} \sqrt{\frac{(n+1) k_{0}}{\tau_{1}}}\right)\right)=0,
$$

and the invariant solution is

$$
y(x)=\alpha \cosh \left(\sqrt{\frac{(n+1) \tau_{1}}{k_{0}}}\left(x-\beta k_{0}\right)\right)^{1 /(n+1)} .
$$

Case $4\left(\tau_{1} \neq 0, \tau_{2} \neq 0\right)$. For the last case, (13) can be rewritten:

$$
y^{\prime \prime} y^{n+1}+n y^{n} y^{\prime 2} k_{0}-y^{n} \tau_{1}-y \tau_{2}=0,
$$

and infinitesimals are

$$
\xi(x, y)=c_{1}, \quad \eta(x, y)=0,
$$

where $c_{1}$ is a constant.

For selection of parameter $c_{1}$ infinitesimals are found:

$$
\xi=1, \quad \eta=0 .
$$

By using (9) we obtain the $\lambda$-symmetry

$$
\lambda=\frac{y^{2} \tau_{1}-y^{1-n} \tau_{2}-k_{0} y^{\prime 2}}{y y^{\prime} k_{0}} .
$$

A solution of (10) is equal to

$$
w\left(x, y, y^{\prime}\right)=\frac{y^{n}}{(n+1) k_{0}}\left(y^{n} y^{\prime 2} k_{0}(n+1)-y^{n+2} \tau_{1} 2 y \tau_{2}\right),
$$

and reduced form is found as

$$
w^{\prime}(x)=0,
$$


in which the general solution is

$$
w(x)=G, \quad G \in \mathbb{R},
$$

and the integrating factor can be written as

$$
\mu=2 y^{n+1} y^{\prime}
$$

Conservation law is

$$
\begin{gathered}
D_{x}\left(\frac { y ( x ) ^ { n } } { k _ { 0 } ( n + 1 ) } \left(y^{\prime}(x) y(x)^{n} k_{0}(n+1)-y(x)^{n+2} \tau_{1}\right.\right. \\
\left.\left.-2 y(x) \tau_{2}\right)\right)=0 .
\end{gathered}
$$

\section{Alternative Approaches for $\lambda$-Symmetries}

5.1. Assuming Linear Form of $\lambda\left(x, y, y^{\prime}\right)$. Let us consider an $n$ th-order ODE:

$$
y^{\prime \prime}=\phi\left(x, y, y^{\prime}\right)
$$

Thus the invariance criteria [14] of (153) is

$$
X^{[\lambda,(n)]}\left(y^{(n)}-\left.f\left(x, y, y^{\prime}, y^{\prime \prime}, \ldots, y^{(n-1)}\right)\right|_{y^{(n)}=f}\right)=0 .
$$

The expansion of relation (154) for $n=2$ gives the determining equation related to fin equation, which are the system of partial differential equations. This system is difficult to solve in terms of lambda function because it is highly nonlinear. For the sake of simplicity $\lambda$ can be assumed to be in a linear form [13-15] such that

$$
\lambda\left(x, y, y^{\prime}\right)=\lambda_{1}(x, y) y^{\prime}+\lambda_{2}(x, y) .
$$

When $v=\partial_{y}, v$ is $\lambda$-symmetry of (1), if and only if

$$
\phi_{y}+\lambda \phi_{y^{\prime}}=A(\lambda)+\lambda^{2}
$$

is satisfied. Applying (2) and (155) to (156) gives

$$
\lambda_{x}+y^{\prime} \lambda_{y}+\phi^{\prime} \lambda_{y^{\prime}}+\lambda^{2}-\phi_{y}-\lambda \phi_{y^{\prime}}=0 .
$$

$\phi$ and $\lambda$ are defined in equation (1). If the equation (155) is substituted in to equation (157), then we obtain determining equation and we find the functions $\lambda_{1}$ and $\lambda_{2}$ from the coefficients of this determining equation. We emphasize that $\lambda$ is a particular solution of (157). If we consider $\phi$ in terms of fin equation and it is substituted into (157), the prolongation formula for the fin equation can be written as

$$
\begin{gathered}
\frac{1}{K(y)^{2}}\left(-y^{\prime 2} K^{\prime}(y)^{2}+H(y)\left(K(y) \lambda_{1}+K^{\prime}(y)\right)\right. \\
+K(y)\left(-H^{\prime}(y)+y^{\prime}\left(y^{\prime} \lambda_{1} K^{\prime}(y)+2 \lambda_{2} K^{\prime}(y)\right.\right. \\
\left.\left.+y^{\prime} K^{\prime \prime}(y)\right)\right) \\
+K(y)^{2}\left(y^{\prime 2} \lambda_{1}^{2}+2 y^{\prime} \lambda_{1} \lambda_{2}+\lambda_{2}^{2}+y^{\prime 2} \lambda_{1_{y}}\right. \\
\left.\left.+y^{\prime} \lambda_{2_{y}}+y^{\prime} \lambda_{1_{x}}+\lambda_{2_{x}}\right)\right)
\end{gathered}
$$$$
=0 \text {. }
$$

By analyzing (158) the coefficient of $y^{\prime 2}$ gives first determining equation such that

$$
\begin{aligned}
& K(y)^{2} \lambda_{1}^{2}+K(y) \lambda_{1} K^{\prime}(y)-K^{\prime}(y)^{2} \\
& +K(y) K^{\prime \prime}(y)+K(y)^{2} \lambda_{1_{y}}=0 .
\end{aligned}
$$

A particular solution of this equation gives $\lambda_{1}$ like this,

$$
\lambda_{1}(x, y)=-\frac{K^{\prime}(y)}{K(y)} \text {. }
$$

Then if we consider coefficient of $y^{\prime}$, we obtain second determining equation as the form

$$
K(y)^{2} \lambda_{2_{y}}+K(y)^{2} \lambda_{1_{x}}=0,
$$

by applying (160) to (161), the general solution of this equation gives $\lambda_{2}$ such that

$$
\lambda_{2}=\beta(x)
$$

The last determining equation becomes

$$
K(y)^{2}\left(\beta(x)^{2}+\beta^{\prime}(x)\right)-K(y) H^{\prime}(y)=0 .
$$

To obtain a simpler form of (163) one can rewrite this equation in the form:

$$
\beta(x)^{2}+\beta^{\prime}(x)-\frac{H^{\prime}(y)}{K(y)}=0 .
$$

In (164), we assume $H^{\prime}(y) / K(y)=a$ in which $a$ is a constant and we write our assumption in (164); we obtain the ordinary differential equation with respect to $\beta$ and so solution of this equation is equal to

$$
\beta(x)=\sqrt{a} \tanh \left(\sqrt{a} x+\sqrt{a} c_{1}\right) .
$$

From the above relation, we find $H^{\prime}(y) / K(y)-a=0$ and if we solve this equation we obtain equation which defines relationship between $K(y)$ and $H(y)$ :

$$
K(y)=\frac{H^{\prime}(y)}{a} .
$$

Now we investigate $\lambda$-symmetries of fin equation for different cases of $H(y)$.

Case $1(H(y)=y)$. It is easy to see that from (166) thermal conductivity $K(y)$ yields

$$
K(y)=\frac{1}{a} .
$$

For this case if $K(y)$ and $H(y)$ are substituted into (160) and (162), respectively, then we find

$$
\lambda_{1}=0, \quad \lambda_{2}=\sqrt{a} \tanh \left(\sqrt{a} x+\sqrt{a} c_{1}\right),
$$


where $c_{1}$ is a constant. And $\lambda$ is obtained by (155)

$$
\lambda\left(x, y, y^{\prime}\right)=\sqrt{a} \tanh \left(\sqrt{a} x+\sqrt{a} c_{1}\right) .
$$

In order to obtain an integrating factor associated to $\lambda$ we must find a first order invariant $w\left(x, y, y^{\prime}\right)$ of $v^{\lambda_{1}}$. And so it is clear that the solution of (10) gives

$$
w\left(x, y, y^{\prime}\right)=c_{1}(x)\left(y^{\prime}-\sqrt{a} y \tanh \left(\sqrt{a} x+\sqrt{a} c_{1}\right)\right) .
$$

If we take $c_{1}(x)=1$ and to write (13) in terms of $\left\{x, w, w^{\prime}\right\}$ we can express the following equality using (170):

$$
y^{\prime}=w(x)+\sqrt{a} y(x) \tanh \left(\sqrt{a} x+\sqrt{a} c_{1}\right) .
$$

Taking derivative of (171) with respect to $x$ gives

$$
y^{\prime \prime}=\sqrt{a} \tanh \left(\sqrt{a} x+\sqrt{a} c_{1}\right) w(x)+a y(x)+w^{\prime}(x),
$$

and by using $y^{\prime}$ and $y^{\prime \prime}(13)$ becomes

$$
w^{\prime}(x)+\sqrt{a} \tanh \left(\sqrt{a} x+\sqrt{a} c_{1}\right) w(x)=0,
$$

and, solving this equation we have

$$
w(x)=G \operatorname{sech}\left(\sqrt{a}\left(c_{1}+x\right)\right), \quad G \in \mathbb{R} .
$$

To find the integrating factor one can write above equation in terms of $G$ :

$$
G=\cosh \left(\sqrt{a}\left(c_{1}+x\right)\right) w .
$$

It is clear that from (12), $\mu$ is obtained in the following form:

$$
\mu=\cosh \left(\sqrt{a}\left(c_{1}+x\right)\right) .
$$

The conserved form of this equation is given by

$$
\begin{aligned}
& D_{x}\left(y^{\prime}(x)-\sqrt{a} y(x) \tanh \left(\sqrt{a}\left(x+c_{1}\right)\right) \cosh \left(\sqrt{a}\left(c_{1}+x\right)\right)\right) \\
& \quad=0
\end{aligned}
$$

which gives the original fin equation. Thus the reduced equation is

$$
y^{\prime}(x)-\sqrt{a} y(x) \tanh \left(\sqrt{a}\left(x+c_{1}\right)\right)-c_{3}=0,
$$

where $c_{3}$ is a constant. Integrating above equation we obtain the solution that satisfies the original equation:

$$
y(x)=c_{2} \cosh \left(\sqrt{a}\left(c_{1}+x\right)\right)+\frac{c_{3} \sinh \left(\sqrt{a}\left(c_{1}+x\right)\right)}{\sqrt{a}},
$$

where $c_{2}$ is a constant.

Case $2\left(H(y)=e^{y}\right)$. From (166) thermal conductivity $K(y)$ can be written as

$$
K(y)=\frac{e^{y}}{a}
$$

By inserting $K(y)$ and $H(y)$ to (161) and (162), respectively, we have

$$
\lambda_{1}=-1, \quad \lambda_{2}=\sqrt{a} \tanh \left(\sqrt{a} x+\sqrt{a} c_{1}\right),
$$

where $c_{1}$ is a constant. From (155) $\lambda$ is equal to

$$
\lambda\left(x, y, y^{\prime}\right)=-y^{\prime}+\sqrt{a} \tanh \left(\sqrt{a} x+\sqrt{a} c_{1}\right) .
$$

The general solution of (10) for this case is given by

$$
w\left(x, y, y^{\prime}\right)=c_{1}(x) e^{y}\left(y^{\prime}-\sqrt{a} \tanh \left(\sqrt{a} x+\sqrt{a} c_{1}\right)\right) .
$$

Now we again use the assumption $c_{1}(x)=1$ and to express (13) in terms of $\left\{x, w, w^{\prime}\right\}$ we can write the following equality using (183):

$$
y^{\prime}=e^{-y}\left(w(x)+\sqrt{a} e^{y} \tanh \left(\sqrt{a} x+\sqrt{a} c_{1}\right)\right) .
$$

Differentiating (184), we obtain $y^{\prime \prime}$

$$
\begin{gathered}
y^{\prime \prime}=e^{-2 y}\left(-\sqrt{a} e^{y} \tanh \left(\sqrt{a} x+\sqrt{a} c_{1}\right) w(x)\right. \\
-w(x)^{2}+e^{y}\left(a e^{y} \operatorname{sech}\left(\sqrt{a}\left(x+c_{1}\right)\right)^{2}\right. \\
\left.\left.+w^{\prime}(x)\right)\right) .
\end{gathered}
$$

If we write $y^{\prime}, y^{\prime \prime}, H(y), K(y)$ in original fin equation (13) then we obtain the same equation (173) such as

$$
w^{\prime}(x)+\sqrt{a} \tanh \left(\sqrt{a} x+\sqrt{a} c_{1}\right) w(x)=0,
$$

and the solution of this equation is given by

$$
w(x)=G \operatorname{sech}\left(\sqrt{a}\left(c_{1}+x\right)\right), \quad G \in \mathbb{R} .
$$

To obtain the integrating factor one can write above equation in terms of $G$ :

$$
G=\cosh \left(\sqrt{a}\left(c_{1}+x\right)\right) w .
$$

It is clear that from (12) that $\mu$ can be derived as

$$
\mu=e^{y}\left(\cosh \left(\sqrt{a}\left(c_{1}+x\right)\right)\right) .
$$

The conserved form of fin equation for this case is

$$
D_{x}\left(y^{\prime}-\sqrt{a} \tanh \left(\sqrt{a}\left(x+c_{1}\right)\right)\right)=0,
$$

which gives the original fin equation. Thus the reduced equation is

$$
y^{\prime}(x)-\sqrt{a} \tanh \left(\sqrt{a}\left(x+c_{1}\right)\right)-c_{3}=0,
$$

where $c_{3}$ is a constant. Solution of this reduced equation which can be called a invariant solution of (13) is

$$
\begin{array}{r}
y(x)=\ln \left(\frac{1}{2} c_{2} \cosh \left(\sqrt{a}\left(c_{1}+x\right)\right)\right. \\
\left.+\frac{c_{3} \sinh \left(\sqrt{a}\left(c_{1}+x\right)\right)}{\sqrt{a}}\right),
\end{array}
$$

where $c_{2}$ is a constant. 
Case $3(H(y)=1 /(m y+n))$. For this selection of $H(y)$, from (166) thermal conductivity $K(y)$ is written by

$$
K(y)=-\frac{m}{a(n+m y)^{2}} .
$$

By using (160) and (162), we obtain

$$
\lambda_{1}=\frac{2 m}{(n+m y)}, \quad \lambda_{2}=\sqrt{a} \tanh \left(\sqrt{a}\left(x+c_{1}\right)\right),
$$

where $c_{1}$ is a constant. And $\lambda$ is obtained by (155):

$$
\begin{aligned}
\lambda\left(x, y, y^{\prime}\right) & =\lambda_{1}(x, y) y^{\prime}+\lambda_{2}(x, y) \\
& =\frac{2 m y^{\prime}}{(n+m y)}+\sqrt{a} \tanh \left(\sqrt{a}\left(x+c_{1}\right)\right) .
\end{aligned}
$$

The general solution of (10) for this case is

$$
\begin{aligned}
w\left(x, y, y^{\prime}\right)=c_{1}(x) & \\
\times & \left(\left(m y^{\prime}+\sqrt{a} n \tanh \left(\sqrt{a}\left(x+c_{1}\right)\right)\right.\right. \\
& \left.+\sqrt{a} m y \tanh \left(\sqrt{a}\left(x+c_{1}\right)\right)\right) \\
& \left.\times\left(m(n+m y)^{2}\right)^{-1}\right) .
\end{aligned}
$$

We have a same assumption $c_{1}(x)=1$ and to define (13) in terms of $\left\{x, w, w^{\prime}\right\}$ we can use following equation:

$$
y^{\prime}=(n+m y)\left(m n w+m^{2} w y-\sqrt{a} \tanh \left(\sqrt{a}\left(x+c_{1}\right)\right)\right) .
$$

Differentiation of $y^{\prime}$ gives $y^{\prime \prime}$ such that

$$
\begin{aligned}
y^{\prime \prime}= & \frac{1}{m}(n+m y) \\
\times & \left(a\left(-\operatorname{sech}\left(\sqrt{a}\left(x+c_{1}\right)\right)^{2}\right)\right. \\
& \left.+\tanh \left(\sqrt{a}\left(x+c_{1}\right)\right)^{2}\right) \\
& \quad-3 \sqrt{a} m \tanh \left(\sqrt{a}\left(x+c_{1}\right)\right) w(x)(n+m y) \\
+ & 2 m^{2} w(x)^{2}(n+m y)^{2} \\
+ & m(n+m y) w(x)^{\prime} .
\end{aligned}
$$

If we substitute $y^{\prime}, y^{\prime \prime}, H(y), K(y)$ in original fin equation (13), we find the following equation:

$$
\begin{aligned}
\operatorname{sech}( & \left.\sqrt{a}\left(x+c_{1}\right)\right)(n+m y)^{2} \\
& \times\left(\sqrt{a} \sinh \left(\sqrt{a}\left(x+c_{1}\right)\right) w(x)\right. \\
& \left.+\cosh \left(\sqrt{a}\left(x+c_{1}\right)\right) w^{\prime}(x)\right)=0,
\end{aligned}
$$

and general solution of (199) yields

$$
w(x)=G \operatorname{sech}\left(\sqrt{a}\left(c_{1}+x\right)\right), \quad G \in \mathbb{R} .
$$

It is clear that from (12) that $\mu$ is obtained in the following form:

$$
\mu=\frac{\cosh \left(\sqrt{a}\left(c_{1}+x\right)\right)}{(n+m y)^{2}} .
$$

The conserved form of fin equation (13) is given by

$$
\begin{aligned}
D_{x}(( & \left(m y^{\prime}+\sqrt{a} n \tanh \left(\sqrt{a}\left(x+c_{1}\right)\right)\right. \\
& \left.\left.+\sqrt{a} m y \tanh \left(\sqrt{a}\left(x+c_{1}\right)\right)\right) \cosh \left(\sqrt{a}\left(c_{1}+x\right)\right)\right) \\
\quad & \left.\left(m(n+m y)^{2}\right)^{-1}\right)=0 .
\end{aligned}
$$

And so we can derive the invariant solution of (13) such as

$$
\begin{aligned}
y(x) & \\
=( & \left(\left(-\sqrt{a} e^{2 \sqrt{a} x}+2 a c_{2}\right) \cosh \left(\sqrt{a}\left(c_{1}+x\right)\right)\right. \\
& +\left(e^{2 \sqrt{a} x}+2 \sqrt{a} c_{2}\right) \\
& \left.\left.\times\left(-c m n+\sqrt{a} \sinh \left(\sqrt{a}\left(c_{1}+x\right)\right)\right)\right)\right) \\
& \times\left(c^{2}\left(e^{2 \sqrt{a} x}+2 \sqrt{a} c_{2}\right)\right)^{-1},
\end{aligned}
$$

where $c_{2}$ is a constant.

5.2. $\lambda$-Symmetries and Jacobi Last Multiplier Approach. The $\lambda$-prolongation of $X(4)$ is

$$
\begin{aligned}
\operatorname{pr} X=\xi & (x, y) \partial_{x}+\eta(x, y) \partial_{y}+\eta^{(1)}\left(x, y, y^{\prime}, y^{\prime \prime}, \ldots, y^{(n-1)}\right) \\
& \times \partial_{y^{\prime}}+\eta^{(2)}\left(x, y, y^{\prime}, y^{\prime \prime}, \ldots, y^{(n-1)}\right) \partial_{y^{\prime \prime}}
\end{aligned}
$$

with

$$
\eta^{(n+1)}=\left[\left(D_{x}+\lambda\right) \eta^{(n)}-y^{\prime}\left(D_{x}+\lambda\right) \xi\right],
$$

where $D_{x}$ is total derivative operator with respect to $x$ such that

$$
D_{x}=\partial_{x}+\sum_{k=0}^{n} y^{(k+1)} \partial_{y^{(k)}}, \quad y^{(0)} \equiv y, \quad \eta^{(0)} \equiv \eta .
$$

In this section we analyze $\lambda$-symmetries of fin equation by using Jacobi Last Multiplier as another approach. First (153) can be written by using system of first order equations, which is equivalent to the expression

$$
w_{i}^{\prime}=W_{i}\left(t, w_{1}, \ldots, w_{n}\right),
$$

the Jacobi Last Multiplier of (207) $M$ is found

$$
\frac{d \log (M)}{d t}+\sum_{i=1}^{n} \frac{\partial W_{i}}{\partial w_{i}}=0,
$$


where, namely, $M$ is

$$
M=\exp \left(-\int \sum_{i=1}^{n} \frac{\partial W_{i}}{\partial w_{i}} d t\right) .
$$

The nonlocal approach [17] to $\lambda$-symmetries is analyzed to seek $\lambda$-symmetries such that

$$
w^{\prime}=\lambda=\sum_{i=1}^{n} \frac{\partial W_{i}}{\partial w_{i}}
$$

With this approach the function is considered in the form such as $w=\log (1 / M)$. However it is a fact that the relation (207) cannot be used if the divergence of (207) Div $\equiv$ $\sum_{i=1}^{n}\left(\partial W_{i} / \partial w_{i}\right)$ is equal to zero. Therefore $w$ has to be chosen in this form because any Jacobi Last Multiplier is a first integral of (207). In this subsection we consider some cases, which we discussed in Section 3 for functions $K(y)$ and $H(y)$ in order to compare $\lambda$-symmetries in terms of different approaches:

5.2.1. $K(y)=k_{0}, H(y)=\tau_{1} y+\tau_{2}$. For this case the divergence of the fin equation (13) yields

$$
\lambda_{j}=0
$$

Substituting $\lambda_{j}$ into (204) then from the solution of the determining equations (154) we obtain eight-parameter $\lambda$ infinitesimals:

$$
\begin{aligned}
& \xi^{(\lambda)}=\frac{1}{2 \tau_{1}} e^{-2 x \sqrt{\tau_{1} / k_{0}}} \times\left(\left(c_{4}+c_{5} e^{4 x \sqrt{\tau_{1} / k_{0}}}\right)+2 e^{x \sqrt{\tau_{1} / k_{0}}}\right. \\
& \times\left(\left(c_{3} e^{x \sqrt{\tau_{1} / k_{0}}}+c_{1} y+c_{2} e^{2 x \sqrt{\tau_{1} / k_{0}}}\right) \tau_{1}\right. \\
&\left.\left.+\left(c_{1}+c_{2} e^{2 x \sqrt{\tau_{1} / k_{0}}}\right) \tau_{2}\right)\right) \\
& \eta^{(\lambda)}=\frac{1}{2 \sqrt{k_{0}}} \tau_{1}^{3 / 2} e^{-2 x \sqrt{\tau_{1} / k_{0}}} \\
& \times\left(\left(-c_{4}+c_{5} e^{4 x \sqrt{\tau_{1} / k_{0}}}\right) k_{0}\left(y \tau_{1}+\tau_{2}\right)\right. \\
&+2 e^{x \sqrt{\tau_{1} / k_{0}}}\left(-c_{1}+c_{2} e^{2 x \sqrt{\tau_{1} / k_{0}}}\right) y \tau_{1} \\
& \times\left(y \tau_{1}+2 \tau_{2}\right)+2 e^{x \sqrt{\tau_{1} / k_{0}}} \sqrt{\tau_{1} k_{0}} \\
& \times\left(\left(c_{7}+c_{8} e^{2 x \sqrt{\tau_{1} / k_{0}}}+c_{8} e^{x \sqrt{\tau_{1} / k_{0}}}\right) \tau_{1}\right. \\
&\left.\left.\quad+c_{6} e^{x \sqrt{\tau_{1} / k_{0}}}\right)\right)
\end{aligned}
$$

where $c_{i}, i=1, \ldots, 8$ are constants. The generators can be written as

$$
\begin{aligned}
& X_{1}^{(\lambda)}=\frac{e^{-x \sqrt{\tau_{1} / k_{0}}}\left(\tau_{1} y+\tau_{2}\right)}{\tau_{1}} \partial_{x}-\frac{e^{-x \sqrt{\tau_{1} / k_{0}}} y\left(\tau_{1} y+\tau_{2}\right)}{\sqrt{\tau_{1} k_{0}}} \partial_{y}, \\
& X_{2}^{(\lambda)}=\frac{e^{x \sqrt{\tau_{1} / k_{0}}}\left(\tau_{1} y+\tau_{2}\right)}{\tau_{1}} \partial_{x}+\frac{e^{x \sqrt{\tau_{1} / k_{0}}} y\left(\tau_{1} y+\tau_{2}\right)}{\sqrt{\tau_{1} k_{0}}} \partial_{y}, \\
& X_{3}^{(\lambda)}=\partial_{x}, \\
& X_{4}^{(\lambda)}=\frac{e^{-2 x \sqrt{\tau_{1} / k_{0}}} k_{0}}{2 \tau_{1}} \partial_{x}-\frac{e^{-2 x \sqrt{\tau_{1} / k_{0}}} \sqrt{k_{0}}\left(\tau_{1} y+\tau_{2}\right)}{2 \tau_{1}^{3 / 2}} \partial_{y}, \\
& X_{5}^{(\lambda)}=\frac{e^{2 x \sqrt{\tau_{1} / k_{0}}} k_{0}}{2 \tau_{1}} \partial_{x}+\frac{e^{2 x \sqrt{\tau_{1} / k_{0}}} \sqrt{k_{0}}\left(\tau_{1} y+\tau_{2}\right)}{2 \tau_{1}^{3 / 2}} \partial_{y}, \\
& X_{6}^{(\lambda)}=\left(y+\frac{\tau_{2}}{\tau_{1}}\right) \partial_{y}, \quad X_{7}^{(\lambda)}=e^{-x \sqrt{\tau_{1} / k_{0}}} \partial_{y}, \\
& X_{8}^{(\lambda)}=e^{x \sqrt{\tau_{1} / k_{0}}} \partial_{y},
\end{aligned}
$$

which corresponds to the classical Lie point symmetries since $\lambda_{j}$ is equal to zero.

5.2.2. $K(y)=k_{0} y, H(y)=\tau_{1} y^{2}+\tau_{2}$. Another special form we consider here is $K(y)=k_{0} y, H(y)=\tau_{1} y^{2}+\tau_{2}$. For this case we obtain the divergence of (13) in the form

$$
\lambda_{j}=\frac{2 y^{\prime}}{y},
$$

and, by substituting $\lambda_{j}$ into the prolongation formula, the $\lambda$ infinitesimals can be found as follows:

$$
\begin{aligned}
\xi^{(\lambda)}=\frac{1}{2 \tau_{1}} e^{-2 x \sqrt{2 \tau_{1} / k_{0}}} y^{2} & \\
\times & \times\left(\left(c_{4}+c_{5} e^{4 x \sqrt{2 \tau_{1} / k_{0}}}\right) k_{0}\right. \\
& +2 e^{x \sqrt{2 \tau_{1} / k_{0}}}\left(\left(c_{3} e^{x \sqrt{2 \tau_{1} / k_{0}}}\right.\right. \\
& \left.\times\left(c_{1}+c_{2} e^{2 x \sqrt{2 \tau_{1} / k_{0}}}\right) y^{2}\right) \tau_{1} \\
& \left.\left.+\left(c_{1}+c_{2} e^{2 \sqrt{2} x \sqrt{2 \tau_{1} / k_{0}}}\right) \tau_{2}\right)\right),
\end{aligned}
$$




$$
\begin{aligned}
\eta^{(\lambda)}=\frac{1}{4 \sqrt{k_{0}}} & \tau_{1}^{3 / 2} e^{-2 x \sqrt{2 \tau_{1} / k_{0}}} \\
\times( & -\sqrt{2}\left(c_{4}-c_{5} e^{4 x \sqrt{2 \tau_{1} / k_{0}}}\right) k_{0} \\
\times & \left(y^{2} \tau_{1}+\tau_{2}\right) \\
\times & 2 \sqrt{2} e^{x \sqrt{2 \tau_{1} / k_{0}}}\left(c_{2} e^{2 x \sqrt{2 \tau_{1} / k_{0}}}-c_{1}\right) y^{2} \tau_{1} \\
\times & \left(y^{2} \tau_{1}+2 \tau_{2}\right) \times 4 e^{x \sqrt{2 \tau_{1} / k_{0}}} \sqrt{k_{0} \tau_{1}} \\
& \times\left(\left(c_{7}+c_{8} e^{2 x \sqrt{2 \tau_{1} / k_{0}}}+c_{6} e^{x \sqrt{2 \tau_{1} / k_{0}}} y^{2}\right)\right. \\
& \left.\left.\times \tau_{1}+c_{6} e^{x \sqrt{2 \tau_{1} / k_{0}}}\right)\right),
\end{aligned}
$$

where $c_{i}, i=1, \ldots, 8$ are constants. And the generators are found as the following form:

$$
\left.X_{1}^{(\lambda)}=\frac{e^{-x \sqrt{2 \tau_{1} / k_{0}}} y^{2}\left(\tau_{1} y^{2}+\tau_{2}\right)}{\tau_{1}} \partial_{x} \quad-(n+1)^{2} x^{3}\left(c_{2}+c_{1} x\right) \tau_{2}^{2}\right),
$$$$
-\frac{e^{-x \sqrt{2\left(\tau_{1} / k_{0}\right)}} y^{3}\left(\tau_{1} y^{2}+2 \tau_{2}\right)}{\sqrt{2 \tau_{1} k_{0}}} \partial_{y},
$$$$
X_{2}^{(\lambda)}=\frac{e^{x \sqrt{2 \tau_{1} / k_{0}}} y^{2}\left(\tau_{1} y^{2}+\tau_{2}\right)}{\tau_{1}} \partial_{x}
$$$$
+\frac{e^{x \sqrt{2 \tau_{1} / k_{0}}} y^{3}\left(\tau_{1} y^{2}+2 \tau_{2}\right)}{\sqrt{2 \tau_{1} k_{0}}} \partial_{y},
$$$$
X_{3}^{(\lambda)}=y^{2} \partial_{x}
$$$$
X_{4}^{(\lambda)}=\frac{e^{-2 x \sqrt{2 \tau_{1} / k_{0}}} y^{2} k_{0}}{2 \tau_{1}} \partial_{x}
$$$$
-\frac{e^{-2 x \sqrt{\tau_{1} / k_{0}}} y \sqrt{k_{0}}\left(\tau_{1} y^{2}+\tau_{2}\right)}{2 \tau_{1}^{3 / 2}} \partial_{y},
$$$$
X_{5}^{(\lambda)}=\frac{e^{2 x \sqrt{2\left(\tau_{1} / k_{0}\right)}} y^{2} k_{0}}{2 \tau_{1}} \partial_{x}
$$$$
+\frac{e^{2 x \sqrt{\tau_{1} / k_{0}}} y \sqrt{k_{0}}\left(\tau_{1} y^{2}+\tau_{2}\right)}{2 \sqrt{2} \tau_{1}^{3 / 2}} \partial_{y},
$$$$
X_{6}^{(\lambda)}=\left(y^{3}+\frac{y \tau_{2}}{\tau_{1}}\right) \partial_{y},
$$$$
X_{7}^{(\lambda)}=e^{-x \sqrt{2 \tau_{1} / k_{0}}} y \partial_{y},
$$$$
X_{8}^{(\lambda)}=e^{x \sqrt{2 \tau_{1} / k_{0}}} y \partial_{y} .
$$

5.2.3. $K(y)=k_{0} y^{n}, H(y)=\tau_{2}$. The divergence of the fin equation yields

$$
\begin{gathered}
\lambda_{j}=\frac{2 n y^{\prime}}{y} \\
\xi^{(\lambda)}=\frac{1}{2 k_{0} y^{2 n}}\left(2 \left(c_{4}+c_{2} y^{n+1}\right.\right. \\
\left.+x\left(c_{5}+c_{1} y^{n+1}+c_{6} x\right)\right) k_{0} \\
\left.\quad-c_{1}(n+1) x^{3} \tau_{2}\right), \\
\eta^{(\lambda)}=\frac{1}{4(n+1) k_{0}^{2}} y^{n} \\
\times\left(2 \left(2 c_{1} y^{2 n+2}+y^{n+1}\right.\right. \\
\times\left(c_{5}+2 c_{3}(n+1)+2 c_{6} x\right) \\
\left.+2(n+1)\left(c_{7}+c_{8} x\right)\right) k_{0}^{2} \\
+(n+1) x\left(6 c_{2} y^{n+1}\right. \\
\left.\quad+x\left(3 c_{5}-2 c_{3}(n+1)+2 c_{6} x\right)\right) k_{0} \tau_{2}
\end{gathered}
$$

where $c_{i}, i=1, \ldots, 8$ are constants. The corresponding new $\lambda$-symmetries are given:

$$
\begin{aligned}
X_{1}^{(\lambda)}= & \frac{e^{-x \sqrt{2 \tau_{1} / k_{0}} y^{2}\left(\tau_{1} y^{2}+\tau_{2}\right)} \partial_{x}}{\tau_{1}} \\
& -\frac{e^{\left.-x \sqrt{2\left(\tau_{1} / k_{0}\right.}\right)} y^{3}\left(\tau_{1} y^{2}+2 \tau_{2}\right)}{\sqrt{2 \tau_{1} k_{0}}} \partial_{y}, \\
X_{2}^{(\lambda)}= & \frac{e^{x \sqrt{2 \tau_{1} / k_{0}}} y^{2}\left(\tau_{1} y^{2}+\tau_{2}\right)}{\tau_{1}} \partial_{x} \\
& +\frac{e^{x \sqrt{2 \tau_{1} / k_{0}}} y^{3}\left(\tau_{1} y^{2}+2 \tau_{2}\right)}{\sqrt{2 \tau_{1} k_{0}}} \partial_{y}, \\
X_{3}^{(\lambda)}= & y^{2} \partial_{x}, \quad \frac{e^{-2 x \sqrt{\tau_{1} / k_{0}}} y \sqrt{k_{0}}\left(\tau_{1} y^{2}+\tau_{2}\right)}{2 \tau_{1}^{3 / 2}} \partial_{y}, \\
X_{4}^{(\lambda)}= & \frac{e^{-2 x \sqrt{2 \tau_{1} / k_{0}}} y^{2} k_{0}}{2 \tau_{1}} \partial_{x} \\
X_{5}^{(\lambda)}= & \frac{e^{2 x \sqrt{2\left(\tau_{1} / k_{0}\right)}} y^{2} k_{0}}{2 \tau_{1}} \partial_{x} \\
& +\frac{e^{2 x \sqrt{\tau_{1} / k_{0}}} y \sqrt{k_{0}}\left(\tau_{1} y^{2}+\tau_{2}\right)}{2 \sqrt{2} \tau_{1}^{3 / 2}} \partial_{y}, \\
X_{6}^{(\lambda)}= & \left(y^{3}+\frac{y \tau_{2}}{\tau_{1}}\right) \partial_{y}, \\
X_{7}^{(\lambda)}= & e^{-x \sqrt{2 \tau_{1} / k_{0}}} y \partial_{y}, \\
X_{8}^{(\lambda)}= & e^{x \sqrt{2 \tau_{1} / k_{0}} y \partial_{y} .}
\end{aligned}
$$


In this section we present some invariant solutions based on Jacobi multiplier approach.

Case 1. For the case $K(y)=k_{0}, H(y)=\tau_{1} y+\tau_{2}$ we can investigate $X_{6}^{\lambda}$ to find the invariant solution of fin equation. The first prolongation of $X_{6}^{\lambda}$ is

$$
\operatorname{pr} X_{6}^{\lambda}=\left(y+\frac{\tau_{2}}{\tau_{1}}\right) \partial_{y}+y^{\prime} \partial_{y^{\prime}}
$$

and the Lagrange equations are

$$
\frac{d x}{0}=\frac{d y}{y+\tau_{2} / \tau_{1}}=\frac{d y^{\prime}}{y^{\prime}},
$$

which give the first-order invariants:

$$
\tilde{x}=x, \quad \tilde{y}=\frac{y^{\prime}}{\tau_{1} y+\tau_{2}} .
$$

Then one can write the first-order equation in the form

$$
\left(\frac{d \tilde{y}}{d \tilde{x}}+\tilde{y}^{2} \tau_{1}\right) k_{0}-1=0,
$$

and the solution of this equation yields

$$
\frac{\tilde{x}}{k_{0}}-\frac{\operatorname{arctanh}\left(\tilde{y} \sqrt{k_{0} \tau_{1}}\right)}{\sqrt{k_{0} \tau_{1}}}=c_{1},
$$

and the first integral is

$$
D_{x}\left(\frac{x}{k_{0}}-\frac{1}{\sqrt{k_{0} \tau_{1}}} \operatorname{arctanh}\left(\frac{y^{\prime}(x) \sqrt{k_{0} \tau_{1}}}{\tau_{1} y(x)+\tau_{2}}\right)\right)=0,
$$

and this equality gives the original fin equation (13). The reduced form of fin equation is

$$
\frac{x}{k_{0}}-\frac{1}{\sqrt{k_{0} \tau_{1}}} \operatorname{arctanh}\left(\frac{y^{\prime}(x) \sqrt{k_{0} \tau_{1}}}{\tau_{1} y(x)+\tau_{2}}\right)-c=0,
$$

in which the solution of (226) is

$$
y(x)=c_{1} \cosh \left(x \sqrt{\frac{\tau_{1}}{k_{0}}}\right)-\frac{\tau_{2}}{\tau_{1}},
$$

where $c_{1}$ and $c$ are constants.

Case 2. As another case $K(y)=k_{0} y, H(y)=\tau_{1} y^{2}+\tau_{2}$ we consider $X_{6}^{\lambda}$ generator, for example, to find the invariant solution of fin equation. The first prolongation of $X_{6}^{\lambda}$ is written:

$$
\operatorname{pr} X_{6}^{\lambda}=\left(y^{3}+\frac{\tau_{2} y}{\tau_{1}}\right) \partial_{y}+\left(\left(y^{2}-\frac{\tau_{2}}{\tau_{1}}\right) y^{\prime}\right) \partial_{y^{\prime}},
$$

and the Lagrange equation is

$$
\frac{d x}{0}=\frac{d y}{\left(y^{3}+\frac{\tau_{2} y}{\tau_{1}}\right)}=\frac{d y^{\prime}}{\left(y^{2}-\frac{\tau_{2}}{\tau_{1}}\right) y^{\prime}},
$$

and the corresponding first order invariants become

$$
\tilde{x}=x, \quad \tilde{y}=\frac{y^{\prime} y}{\tau_{1} y^{2}+\tau_{2}} .
$$

Thus, the corresponding first-order equation is

$$
\left(\frac{d \tilde{y}}{d \tilde{x}}+2 \tilde{y}^{2} \tau_{1}\right) k_{0}-1=0
$$

and the solution of (231) is

$$
\frac{x}{k_{0}}-\frac{1}{\sqrt{2 k_{0} \tau_{1}}} \operatorname{arctanh}\left(\frac{y^{\prime}(x) y(x) \sqrt{2 k_{0} \tau_{1}}}{\tau_{1} y(x)^{2}+\tau_{2}}\right)=c_{1},
$$

and the first integral is

$$
D_{x}\left(\frac{x}{k_{0}}-\frac{1}{\sqrt{2 k_{0} \tau_{1}}} \operatorname{arctanh}\left(\frac{y^{\prime}(x) y(x) \sqrt{2 k_{0} \tau_{1}}}{\tau_{1} y(x)^{2}+\tau_{2}}\right)\right)=0,
$$

which is equal to the original fin equation (13). The new reduced form is

$$
\frac{x}{k_{0}}-\frac{1}{\sqrt{2 k_{0} \tau_{1}}} \operatorname{arctanh}\left(\frac{y^{\prime}(x) y(x) \sqrt{2 k_{0} \tau_{1}}}{\tau_{1} y(x)^{2}+\tau_{2}}\right)-c=0,
$$

and the solution of (234) is

$$
y(x)=\mp \frac{1}{\sqrt{\tau_{1}}} \sqrt{e^{2 c_{1} \tau_{1}} \cosh \left(\frac{\sqrt{2 \tau_{1}} x}{\sqrt{k_{0}}}\right)-\tau_{2}}
$$

where $c_{1}$ and $c$ are constants.

\section{Concluding Remarks}

This study focuses on applications of different symmetry approaches such as classical Lie point and $\lambda$-symmetries for the one-dimensional steady-state fin problem. We consider both the thermal conductivity and the heat transfer coefficient to be arbitrary functions of temperature. Firstly we concern with determining equations of fin problem to determine infinitesimals functions. The solutions of these determining equations enable us determine various forms of the heat transfer coefficient functions $H(y)$ corresponding to different cases of thermal conductivity function $K(y)$. And so we obtain a lot of cases to investigate in detail and then Lie point symmetries of fin equation are examined. By making use of these Lie point symmetries we determine $\lambda$ symmetries, conservation laws, and new reduced form of fin equation algorithmically.

Another part of this study consists of investigation of $\lambda$ symmetries, in which $\lambda$-function is assumed to be in linear form for simplicity since the corresponding determining equations are highly nonlinear and difficult to solve. This approach is also provided to illustrate new forms of $K(y)$ and $H(y)$ functions for new conservation forms of nonlinear fin equation. As a new approach to $\lambda$-symmetry concept 
the Jacobi Last Multiplier method is discussed and applied in this study. This method has some differences from previous methods mentioned here. In this approach we can use divergence of the equation to construct the prolongation of the same equation and as a result new $\lambda$-symmetries are achieved. So these symmetries are compared with each other. In particular, the comparison is made between Lie point and $\lambda$-symmetry approach with Jacobi last multiplier method. One can say that if Lie point symmetries of given equation are trivial and then this method has advantage to obtain new lambda symmetries. Moreover some invariant solutions can be obtained by using this approach.

\section{Conflict of Interests}

The authors declare that there is no conflict of interests regarding the publication of this paper.

\section{References}

[1] M. Pakdemirli and A. Z. Sahin, "Similarity analysis of a nonlinear fin equation," Applied Mathematics Letters, vol. 19, no. 4, pp. 378-384, 2006.

[2] A. H. Bokhari, A. H. Kara, and F. D. Zaman, "A note on a symmetry analysis and exact solutions of a nonlinear fin equation," Applied Mathematics Letters, vol. 19, no. 12, pp. 13561360, 2006.

[3] O. O. Vaneeva, A. G. Johnpillai, R. O. Popovych, and C. Sophocleous, "Group analysis of nonlinear fin equations," Applied Mathematics Letters, vol. 21, no. 3, pp. 248-253, 2008.

[4] G. W. Bluman and S. Kumei, Symmetries and Differential Equations, Springer, New York, NY, USA, 1989.

[5] L. V. Ovsiannikov, Group Analysis of Differential Equations, Nauka, Moscow, Russia, 1978.

[6] N. H. Ibragimov, Ed., CRC Handbook of Lie Group Analysis of Differential Equations, Volumes I-III, CRC Press, 1994.

[7] P. J. Olver, Applications of Lie Groups to Differential Equations, vol. 107 of Graduate Texts in Mathematics, Springer, New York, NY, USA, 1986.

[8] H. Stephani, Diff erential Equations: Their Solutions Using Symmetries, Cambridge University Press, Cambridge, UK, 1989.

[9] T. Özer, "Symmetry group classification for one-dimensional elastodynamics problems in nonlocal elasticity," Mechanics Research Communications, vol. 30, no. 6, pp. 539-546, 2003.

[10] T. Özer, "On symmetry group properties and general similarity forms of the Benney equations in the Lagrangian variables," Journal of Computational and Applied Mathematics, vol. 169, no. 2, pp. 297-313, 2004.

[11] T. Özer, "Symmetry group classification for two-dimensional elastodynamics problems in nonlocal elasticity," International Journal of Engineering Science, vol. 41, no. 18, pp. 2193-2211, 2003.

[12] S. Kim, J. Moon, and C. H. Huang, "An approximate solution of the nonlinear fin problem with temperature-dependent thermal conductivity and heat transfer coefficient," Journal of Physics D: Applied Physics, vol. 40, no. 14, article 038, pp. 4382-4389, 2007.

[13] C. Muriel and J. L. Romero, "First integrals, integrating factors and $\lambda$-symmetries of second-order differential equations," Journal of Physics A, vol. 42, no. 36, Article ID 365207, 17 pages, 2009.
[14] C. Muriel and J. L. Romero, "New methods of reduction for ordinary differential equations," IMA Journal of Applied Mathematics, vol. 66, no. 2, pp. 111-125, 2001.

[15] C. Muriel and J. L. Romero, "Integrating factors and $\lambda$ symmetries," Journal of Nonlinear Mathematical Physics, vol. 15, supplement 3, pp. 300-309, 2008.

[16] C. Muriel and J. L. Romero, " $\mathrm{C}^{\infty}$ symmetries and reduction of equations without lie point symmetries," Journal of Nonlinear Mathematical Physics, vol. 13, no. 1, pp. 167-188, 2003.

[17] M. C. Nucci and D. Levi, " $\lambda$-symmetries and Jacobi last multiplier," Nonlinear Analysis. Real World Applications, vol. 14, no. 2, pp. 1092-1101, 2013. 


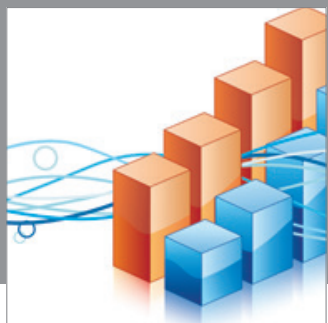

Advances in

Operations Research

mansans

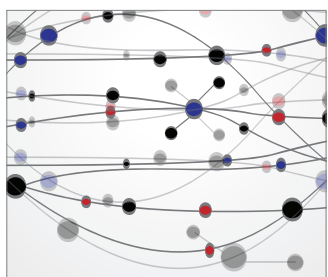

The Scientific World Journal
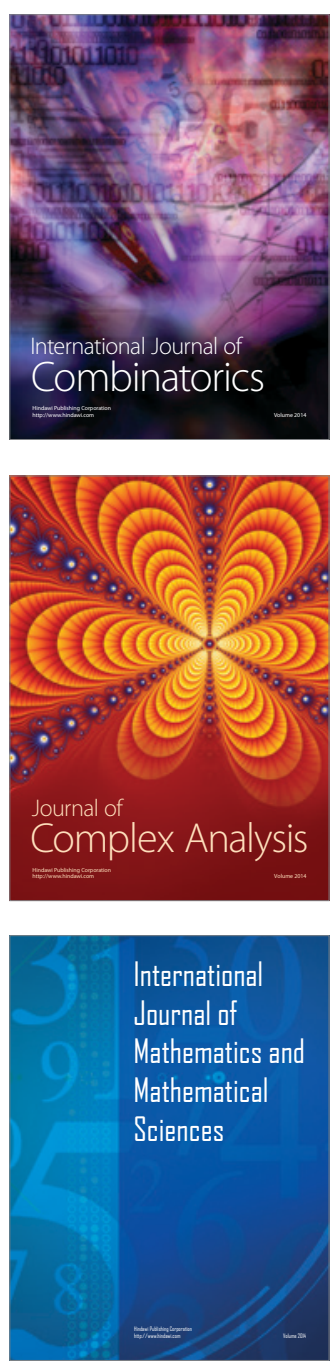
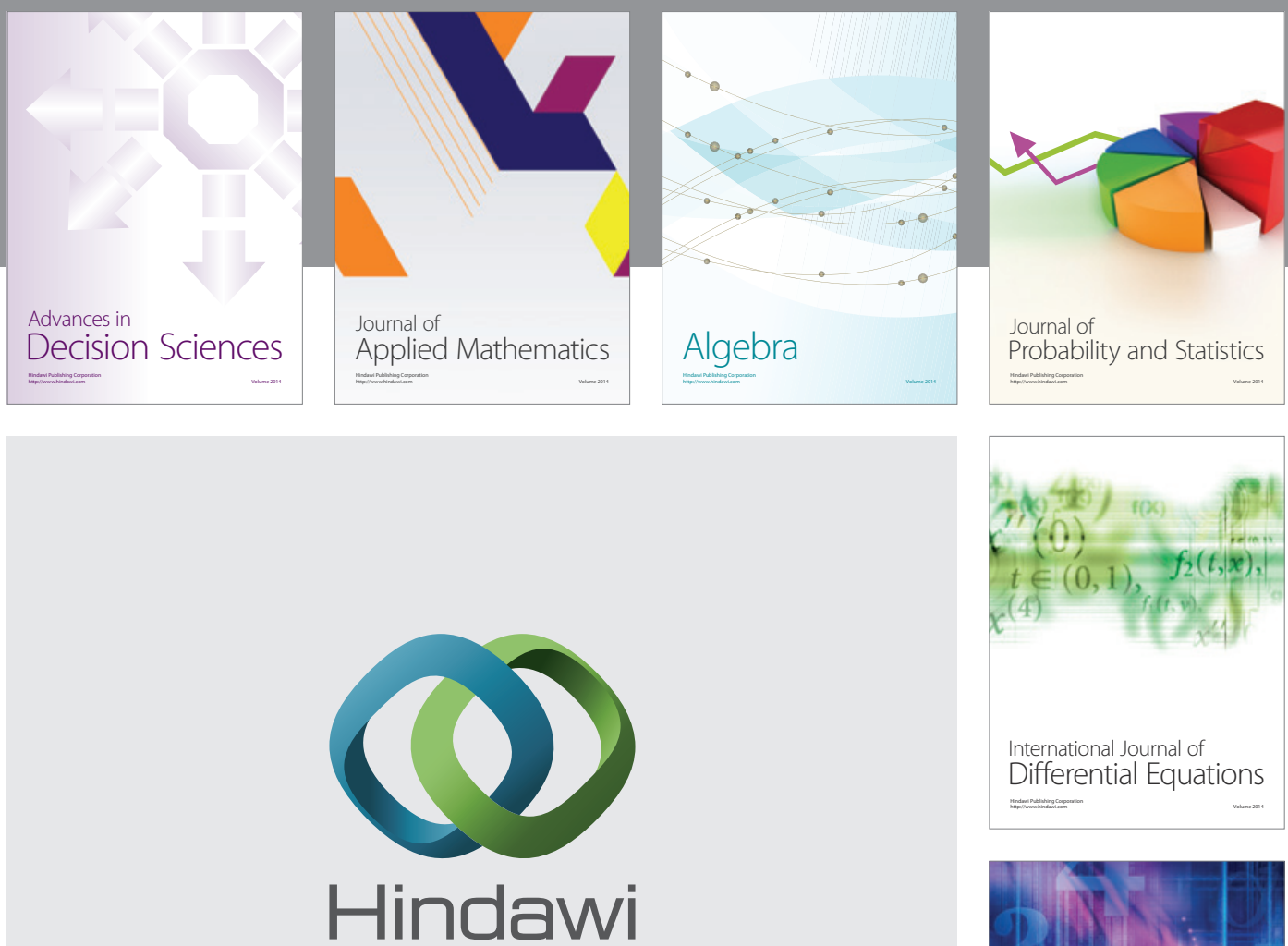

Submit your manuscripts at http://www.hindawi.com
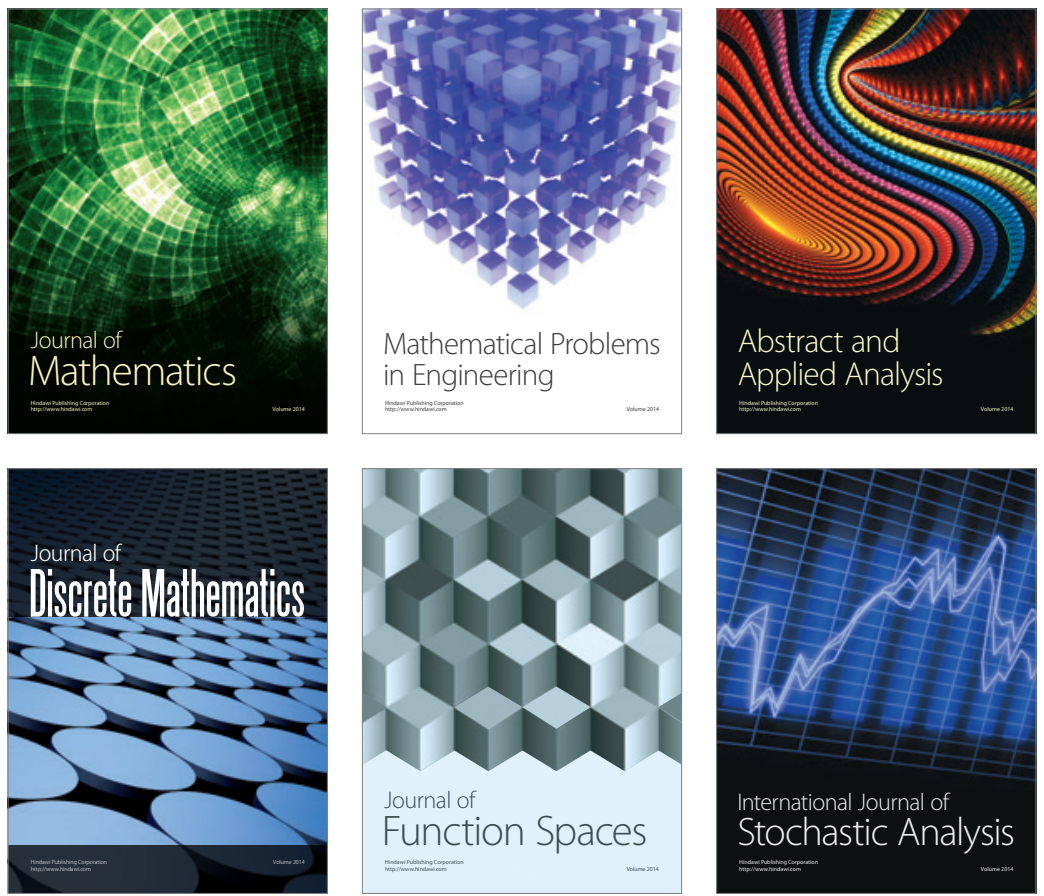

Journal of

Function Spaces

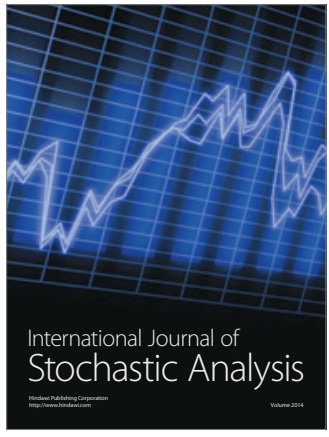

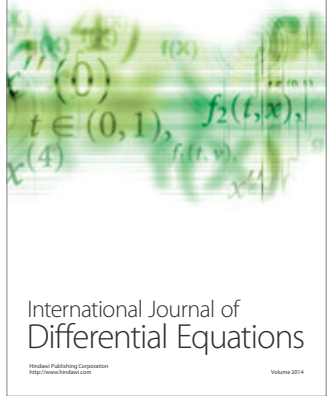
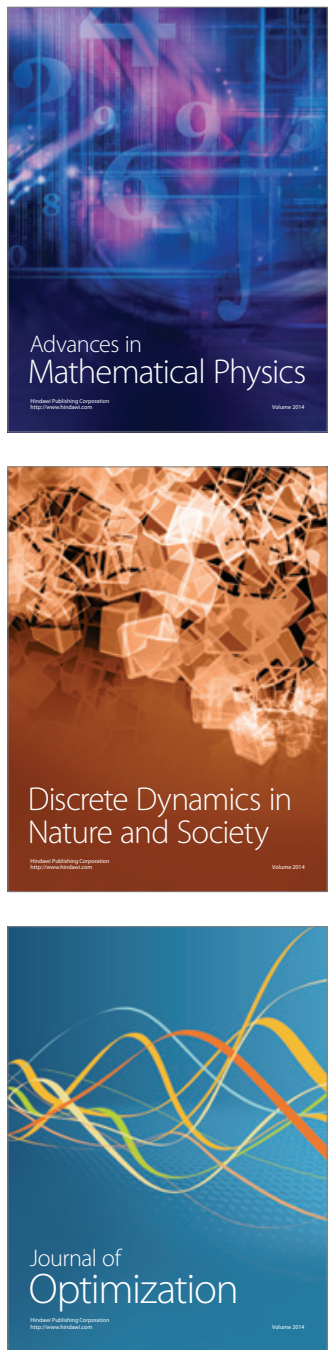